\title{
A network approach to risk theory and portfolio selection
}

\author{
Roy Cerqueti and Claudio Lupi
}

\begin{abstract}
In the context of portfolio theory, the evaluation of risk is of paramount relevance. In this respect, the connections among the risky assets of the portfolio should be carefully explored. This paper elaborates on this topic. We define a portfolio through a network, whose nodes are the assets composing it. The weights on the nodes and the arcs represent the share of capital invested on the assets and the dependence among them, respectively. The risk profile of the portfolio will be given through a suitably defined risk measure on the portfolio-network. The standard Markowitz theory will be rewritten in this particular setting. Surprisingly, we will note that the resulting decision problem is not consistent with an adapted version of the axiomatization of the standard expected utility theory.
\end{abstract}

\section{Introduction}

Since its inception in [7], portfolio theory has attracted the academic debate not only for its potential applications, but also for the space left for theoretical improvements. The original model is grounded on several restrictive assumptions such as uniperiodal setting, Gaussian returns, risks measured by variances and expected returns represented by historical means, absence of transaction costs and of default risks. In the following years, Markowitz's framework has been extended under several respects. One of the most notable improvements of the original formulation can be found in $[9,10]$, where continuous time has been introduced, and in [13], where the model has been extended by introducing a multiperiodal setting. Among recent

Roy Cerqueti

University of Macerata, Department of Economics and Law. Via Crescimbeni, 20. I-62100 Macerata, Italy. Tel.: +39 07332583246 - Fax: +39 0733258 3205. e-mail: roy.cerqueti@unimc.it

Claudio Lupi

University of Molise, Department of Economics. Via F. De Sanctis, I-86100 Campobasso, Italy. Tel.: +390874 404451 - Fax: +390874 311124 e-mail: lupi@unimol.it 
extensions, it is interesting for this paper to mention [5], that provides the reinterpretation of the mean-variance model in the context of semi-copulas; [11] adopts a topological perspective for highlighting the relationship between portfolio and Euclidean distance in the Markowitz model; [2,3] present a mixed discrete-continuous time mean-variance model in the presence of infrequently traded assets. An extensive survey on sixty years of research on the mean-variance model has been outlined by its pioneer (see [8]).

In this paper we further proceed along this research path and provide an extension of the Markowitz model by including in the model formulation the structure of the connections among the assets. For this purpose, we move from [4] and identify portfolios with networks, whose nodes are the assets. ${ }^{1}$

Furthermore, we discuss the consistency of the Markowitz theory with the axiomatization of the expected utility. We adopt the theoretical framework developed in [4], and argue that the goodness of a risk-minimization criterion for ordering preferences should be not necessarily in accord to expected utility. In so doing, we are exactly in line with the philosophical proposal of [1], who show that commonly used risk acceptance criteria do not agree with expected utility.

The original contributions of the paper are the following: first, we present a novel formulation of a classical portfolio problem in the language of networks, hence including explicitly the connections among the risky assets; second, we adopt a recent axiomatization of the expected utility theory in the language of the networks (see [4]) and show that there exists a parametrization of the mean-variance utility function which is not consistent with such a parametrization.

In general, we feel in line with [6], who discuss risk minimization problems by explicitly modelling also the topological and the stochastic dependence structure of the involved decision variables. In particular, to the best of our knowledge, this is the first attempt to model Markowitz theory in the language of networks.

The rest of the paper is organized as follows: the next section introduces the portfolios we deal with. Section 3 outlines the concept of risk measure, along with a needed equivalence relation over the set of portfolios. Section 4 contains the reinterpretation of Markowitz's mean-variance utility theory in our specific setting, and the analysis of the consistency of the associated preference criterion with expected utility. The last section offers some concluding remarks. To be as self-contained as possible, we have reformulated the expected utility axiomatization presented in [4] in our framework. Such a reformulation is contained in the Appendix.

\section{The set of portfolios}

We propose here a very general setting of a portfolio model: such a general setting will then be adapted to the Markowitz model.

\footnotetext{
${ }^{1}$ For a survey on networks, see [14, 12].
} 


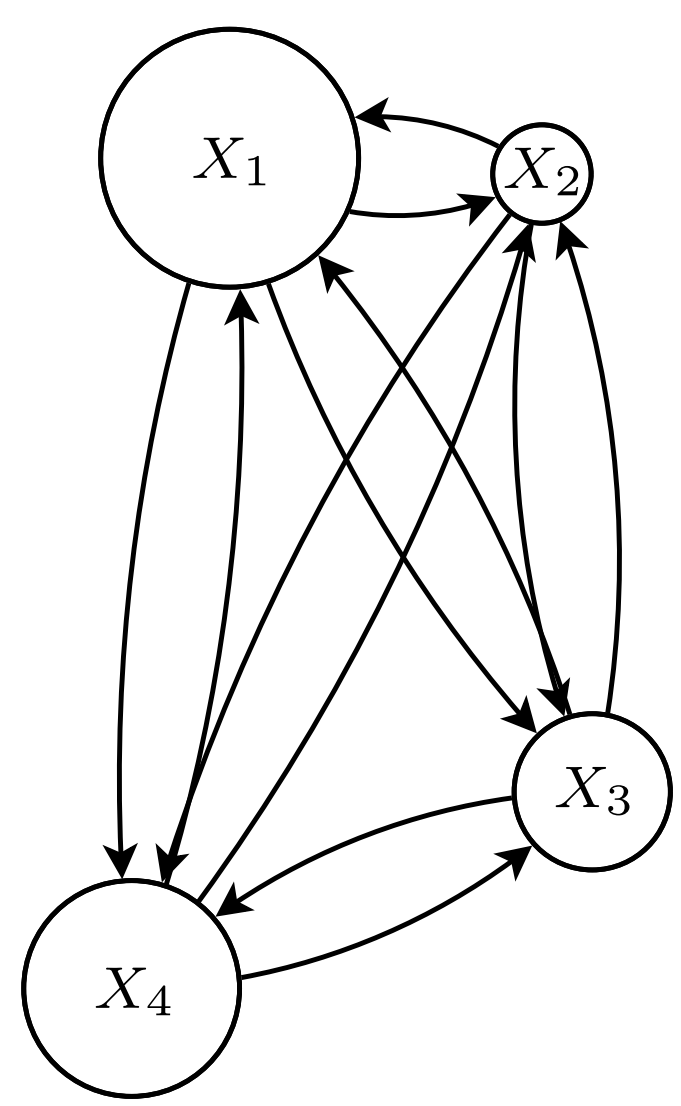

Fig. 1 Network representation of a four-asset portfolio. The size of each node is proportional to $\rho_{j}$; the length $\left(\delta_{i \rightarrow j}\right)$ of each arc is inversely proportional to the dependence among the assets; $v(i, j)=1 \forall i \neq j$.

First, we introduce a probability space $(\Omega, \mathscr{F}, \mathbb{P})$ for all the random variables used in this paper. These random variables are collected in the set $\mathscr{A}$.

Portfolios are assumed to be formed by $n$ assets, whose random returns are the elements of a subset $\mathscr{S}$ of $\mathscr{A}$ as follows:

$$
\mathscr{S}=\left\{X_{1}, X_{2}, \ldots, X_{n}\right\} \subseteq \mathscr{A} .
$$

The set $\mathscr{S}$ is identified as the set of the nodes (vertices) of a weighted oriented graph (see Figure 1).

Weights of nodes and arcs are formalized as follows: 
- we define a function $\rho: \mathscr{S} \rightarrow \mathbb{R}$ such that $\rho\left(X_{j}\right)=\rho_{j}$ represents the weight of $X_{j}$, for each $j=1,2, \ldots, n$;

- we introduce a binary variable which certifies the existence of an arc:

$$
v(i, j)=\left\{\begin{array}{l}
1, \text { if there exists the oriented arc connecting } i \text { and } j, \\
0, \text { otherwise; }
\end{array}\right.
$$

for each $i, j=1,2, \ldots, n$;

- we introduce a real number $\delta_{i \rightarrow j} \in \mathbb{R}$ representing the weight of the oriented arc from $X_{i}$ to $X_{j}$, for each $i, j=1,2, \ldots, n, i \neq j$.

A portfolio $\pi$ is then formalized by the knowledge of its nodes and of functions $\rho$ 's, $v$ 's and $\delta$ 's, so that:

$$
\pi=(\mathscr{S}, \rho, v, \delta),
$$

where $\rho=\left\{\rho_{j}\right\}_{j=1,2, \ldots, n}, v=\{v(i, j)\}_{i, j=1,2, \ldots, n}$ and $\delta=\left\{\delta_{i \rightarrow j}\right\}_{i, j=1,2, \ldots, n}$.

The space of the portfolios is then given by

$$
\mathbf{P}=\left\{(\mathscr{S}, \rho, v, \delta) \in \mathscr{P}(\mathscr{A}) \times \mathbb{R}^{|\mathscr{S}|} \times\{0,1\}^{|\mathscr{S}|^{2}} \times \mathbb{R}^{|\mathscr{S}|^{2}}\right\}
$$

where $\mathscr{P}(\star)$ is the usual notation for the set of the parts of $\star$.

The presence of the shares of capital invested in the assets of $\mathscr{S}$ is implicitly included in the definition of the weights $\rho$ 's and $\delta$ 's. We will see this in details while discussing the Markowitz model.

\section{Risk measure of portfolio}

In order to define risk measures, we need to introduce the concept of equivalence of portfolios. Indeed, as we will see, a consistent risk measure should assign the same value to portfolios with some specific characteristics.

We adopt the framework of [4], here adapted to our specific context.

Definition 1. Consider two portfolios

$$
\pi_{k}=\left(\mathscr{S}^{(k)}, \rho^{(k)}, v^{(k)}, \delta^{(k)}\right) \in \mathbf{P}, \quad k=1,2 .
$$

We say that portfolio $\pi_{1}$ is equivalent to portfolio $\pi_{2}-$ i.e.: $\pi_{1} \equiv \pi_{2}-$ when one of the following conditions is satisfied:

(i) $\left(\mathscr{S}^{(1)}, \rho^{(1)}, v^{(1)}, \delta^{(1)}\right)=\left(\mathscr{S}^{(2)}, \rho^{(2)}, v^{(2)}, \delta^{(2)}\right)$;

(ii) if $1=v^{\left(k_{1}\right)}(i, j) \neq v^{\left(k_{2}\right)}(i, j)=0$, then $\delta_{i \rightarrow j}^{\left(k_{1}\right)}=0$, for each $i, j$ indices of the nodes of $\mathscr{S}^{\left(k_{1}\right)}$ and $k_{1}, k_{2}=1,2$ with $k_{1} \neq k_{2}$, all other things being equal; 
(iii) if $X_{j} \in \mathscr{S}^{\left(k_{1}\right)} \backslash \mathscr{S}^{\left(k_{2}\right)}$, then $\rho_{j}^{\left(k_{1}\right)}=0$ and $\delta_{i \rightarrow j}^{\left(k_{1}\right)}=\delta_{j \rightarrow i}^{\left(k_{1}\right)}=0$, for each $i$ index of the nodes in $\mathscr{S}^{\left(k_{1}\right)} \cup \mathscr{S}^{\left(k_{2}\right)}$ and $k_{1}, k_{2}=1,2$ with $k_{1} \neq k_{2}$, all other things being equal.

Definition 1 has a very relevant interpretation. In fact, once a portfolio $\pi$ is selected, then it is possible to create another portfolio $\tilde{\pi}$ which is equivalent to $\pi$. To do this, one can add other assets, labeled with $j_{1}, \ldots, j_{k}$. Any new asset must be associated to null weights, so that $\rho_{j}=0$ and $\delta_{i \rightarrow j}=\delta_{j \rightarrow i}=0$, for each $j=j_{1}, \ldots, j_{k}$ and for each $i$-th node of $\tilde{\pi}$. The equivalence is then of paramount relevance, because it allows us to consider homogeneous portfolios sharing the same set of nodes. Furthermore, under the condition of imposing some further null weights, it is also not restrictive to assume that all the couples of assets of the portfolio are associated to two oriented arcs. Indeed, it is equivalent to consider $v(i, j)=0$ or, alternatively, $v(i, j)=1$ and $\delta_{i \rightarrow j}=\delta_{j \rightarrow i}=0$.

In order to deal with the elements of the set $\mathbf{P}$, we need to introduce the operators allowing to combine portfolios. In particular, the following definitions formalize the direct sum of two portfolios and the product of a portfolio for a scalar.

Definition 2. Consider $\pi_{1}, \pi_{2} \in \mathbf{P}$ such that $\pi_{k} \equiv\left(\mathscr{S}^{(k)}, \rho^{(k)}, v^{(k)}, \boldsymbol{\delta}^{(k)}\right)$ for $k=1,2$. The direct sum of $\pi_{1}$ and $\pi_{2}$ is

$$
\pi=\pi_{1} \oplus \pi_{2} \in \mathbf{P}
$$

where $\pi \equiv(\mathscr{S}, \rho, v, \delta)$ with $\mathscr{S}=\mathscr{S}^{(1)} \cup \mathscr{S}^{(2)}$ and, for each $i, j=1, \ldots, n$, it is $\rho_{j}=\rho_{j}^{(1)}+\rho_{j}^{(2)}, \delta_{i \rightarrow j}=\delta_{i \rightarrow j}^{(1)}+\delta_{i \rightarrow j}^{(2)}$ and $v(i, j)=\max \left\{v^{(1)}(i, j), v^{(2)}(i, j)\right\}$.

Definition 3. Consider $\pi \equiv(\mathscr{S}, \rho, v, \delta) \in \mathbf{P}$ and a scalar $\alpha \in \mathbb{R}$.

The product scalar-network $\alpha \cdot \pi$ is a new network $\pi_{\alpha} \equiv\left(\mathscr{S}, \rho^{(\alpha)}, v, \delta^{(\alpha)}\right)$ such that $\rho_{j}^{(\alpha)}=\alpha \cdot \rho_{j}$ and $\delta_{i \rightarrow j}^{(\alpha)}=\alpha \cdot \delta_{i \rightarrow j}$, for each $i, j=1, \ldots, n$.

The topological structure of the set $\mathbf{P}$ - endowed with the binary operator $\oplus$ assures that the set of portfolios is quite rich. We refer to [4] for an extensive analysis of this aspect.

We maintain the definition of risk measure of a portfolio as general as possible.

Definition 4. A risk measure of a portfolio is a function $\mu: \mathbf{P} \rightarrow \mathbb{R}$ such that:

- If $\pi_{1} \equiv \pi_{2}$, then $\mu\left(\pi_{1}\right)=\mu\left(\pi_{2}\right)$;

- $\mu$ provides a total order $\preceq \mu$ over the set $\mathbf{P}$ as follows:

$$
\pi_{1} \preceq_{\mu} \pi_{2} \quad \text { if and only if } \quad \mu\left(\pi_{1}\right) \leq \mu\left(\pi_{2}\right)
$$

for each $\pi_{1}, \pi_{2} \in \mathbf{P}$

- $\mu\left(\pi_{1}\right)<\mu\left(\pi_{2}\right)$ means that $\pi_{1}$ is less risky — in the sense of $\preceq \mu$ - than $\pi_{2}$.

In [4] the interested reader can find a detailed discussion of this type of risk measures, along with some examples in the general context of networks. Moreover, the 
quoted paper contains also the analysis of the consistency of this kind of risk measures with respect to a reformulation of the expected utility theory: for completeness, we offer in the Appendix a brief account of the main results.

It is important to note that the risk measures of a portfolio could be viewed as equivalence classes rather than single elements. This aspect will be of remarkable relevance in the discussion of our variant of the Markowitz model and the consistency of the associated preference order with the expected utility axioms (see the next section). Thus, we here present a concept of equivalence for risk measures of portfolios, in agreement with the general scheme proposed in [4]:

Definition 5. Consider two risk measures of portfolios $\mu_{k}: \mathbf{P} \rightarrow \mathbb{R}$, with $k=1,2$. $\mu_{1}$ is equivalent to $\mu_{2}-$ and we indicate $\mu_{1} \equiv \mu_{2}$ - if and only if

$$
\mu_{1}\left(\pi_{1}\right) \leq \mu_{1}\left(\pi_{2}\right) \quad \text { if and only if } \quad \mu_{2}\left(\pi_{1}\right) \leq \mu_{2}\left(\pi_{2}\right),
$$

for each $\pi_{1}, \pi_{2} \in \mathbf{P}$.

Notice that the equivalence instituted by Definition 5 involves how portfolios are ranked by the risk measure, and not how they are objectively evaluated. Substantially, equivalence is associated to the order of the portfolios, even in presence of very different scales of evaluation.

\section{Rewriting the Markowitz model}

This section is devoted to the proposal of a rewriting of the standard Markowitz model. The aim is twofold: first, we pursue the scope of applying the network theoretical framework presented above to the classical portfolio theory; second, we show that risk measures of portfolios in Markowitz theory could also be not consistent with the expected utility axiomatization. More than this, we show that two equivalent measures could behave differently in this respect. Such a finding suggests a substantial independence of the family of risk acceptance criteria with the axiomatization of Von Neumann and Morgerstern.

Suppose that the shares of capital invested on $X_{1}, X_{2}, \ldots, X_{n}$ are $x_{1}, x_{2}, \ldots, x_{n}$, respectively.

The general formulation of the optimal portfolio problem is:

$$
\begin{cases}\min _{x_{1}, \ldots, x_{n}} & \mu(\pi) ; \\ \text { s.t. }, \quad \sum_{j=1}^{n} x_{j}=1,\end{cases}
$$

where $\mu$ is the risk measures of portfolios. See below for two proposals for $\mu$. 


\subsection{First model}

Moving from the definition of portfolios provided in Section 2, we identify the weights associated to Markowitz's mean-variance utility case.

The weights of the (oriented) arcs are given by:

$$
\delta_{i \rightarrow j}=\delta_{j \rightarrow i}=x_{i} x_{j} \mathbb{C}\left[X_{i}, X_{j}\right], \quad \forall i, j=1, \ldots, n, i \neq j,
$$

where $\mathbb{C}$ is the covariance operator. If $i=j$, then

$$
\delta_{j \rightarrow j}=x_{j} \sqrt{\mathbb{V}\left[X_{j}\right]}
$$

The binary variables are redundant, in the sense that $v(i, j)=1$ for each $i, j=$ $1, \ldots, n$.

Functions $\rho$ 's are given by expected values of the returns of the assets weighted for the related shares of the portfolio, so that:

$$
\rho_{j}=x_{j} \mathbb{E}\left[X_{j}\right], \quad \forall j=1, \ldots, n .
$$

The mean-variance utility can be written as (is equivalent to) $\mu: \mathbf{P} \rightarrow \mathbb{R}$ such that:

$$
\mu_{1}(\pi) \equiv-\sum_{j=1}^{n} \rho_{j}+a \cdot\left\{\sum_{j=1}^{n} v(j, j)\left[\delta_{j \rightarrow j}\right]^{2}+2 \sum_{i<j} v(i, j) \delta_{i \rightarrow j}\right\}
$$

\subsection{Second model}

In this case, the weights of the (oriented) arcs are given by:

$$
\delta_{i \rightarrow j}=\delta_{j \rightarrow i}=x_{i} x_{j} \mathbb{C}\left[X_{i}, X_{j}\right], \quad \forall i, j=1, \ldots, n, i \neq j,
$$

where $\mathbb{C}$ is the covariance operator. If $i=j$, then

$$
\delta_{j \rightarrow j}=x_{j}^{2} \mathbb{V}\left[X_{j}\right]
$$

As in the previous case, $v(i, j)=1$ for each $i, j=1, \ldots, n$.

Functions $\rho$ 's are given by expected values of the returns of the assets weighted for the related shares of the portfolio, so that:

$$
\rho_{j}=x_{j} \mathbb{E}\left[X_{j}\right], \quad \forall j=1, \ldots, n .
$$

The mean-variance utility can be written as (is equivalent to) $\mu: \mathbf{P} \rightarrow \mathbb{R}$ such that:

$$
\mu_{2}(\pi) \equiv-\sum_{j=1}^{n} \rho_{j}+a \cdot\left\{\sum_{j=1}^{n} v(j, j) \delta_{j \rightarrow j}+2 \sum_{i<j} v(i, j) \delta_{i \rightarrow j}\right\} .
$$




\subsection{Some results}

It is easy to check that the following proposition is true:

Proposition 1. The risk measures of portfolios $\mu_{1}$ and $\mu_{2}$ are equivalent.

Observe that the preference order induced by $\mu_{1}: \mathbf{P} \rightarrow \mathbb{R}$ in (4) is not consistent with the expected utility theory. This is true by [4, Proposition 3], since $\mu_{1}$ is nonlinear with respect to $\delta_{i \rightarrow j}$ and depends also on $\rho_{j}$.

On the contrary, a simple computation gives that $\mu_{2}: \mathbf{P} \rightarrow \mathbb{R}$ in (5) is consistent with the expected utility axiomatization.

\section{Conclusions}

In this paper we present a rewriting of the classical mean-variance portfolio theory in the context of networks. With this aim, we have proposed a definition of the set of portfolios in the context of networks. The operators acting on such a set have been introduced, to allow the analysis of the portfolio theory in this specific context. Moreover, we have developed two portfolio models and created a suitable risk measure on portfolios. One of the considered risk measure is not consistent with the expected utility axiomatization, as reinterpreted by [4]. This outcome is in line with a recent strand of literature (see $[1,4]$ ), and suggests further developments to classify in this respect the main risk measures used in portfolio theory.

\section{Appendix: A reformulation of the expected utility axiomatization}

[4] offer a reformulation of the standard expected utility axiomatization as proposed, e.g., by [1]. In this Appendix we collect for completeness the expected utility axioms expressed in terms of networks - which are now our portfolios — as proposed in [4, Section 4].

1. Weak order — Preferences are: 1) complete, i.e. the decider can state whether two portfolios are equivalent or whether one is preferred to the other; 2) transitive, i.e. given three portfolios $\pi_{1}, \pi_{2}, \pi_{3}$, if $\pi_{1}$ is preferred to $\pi_{2}$ and $\pi_{2}$ is preferred to $\pi_{3}$, then $\pi_{1}$ is preferred to $\mathbf{N}_{3} ; 3$ ) reflexive, i.e. the decision-maker is indifferent between two equivalent portfolios.

2. Continuity - Given three different portfolios $\pi_{1}, \pi_{2}, \pi_{3}$ such that $\pi_{1}$ is preferred to $\pi_{2}$ and $\pi_{2}$ is preferred to $\pi_{3}$, then there exists a number $p \in(0,1]$ such that the decider is indifferent between the compounded portfolio $p \cdot \pi_{1} \oplus(1-$ $p) \cdot \pi_{3}$ and $\pi_{2}$. 
3. Preference increasing with probabilities (and with connections and weights) - Consider $\mathscr{S}^{(1)}=\left\{X_{1}^{(1)}, X_{2}\right\}$ and $\mathscr{S}^{(2)}=\left\{X_{1}^{(2)}, X_{2}\right\}$, where $X_{1}^{(1)}, X_{1}^{(2)}, X_{2} \in$ $\mathscr{A}$ and $X_{1}^{(1)}>_{S D 1} X_{2}^{(2)}$, where $>_{S D 1}$ denotes stochastic dominance of order 1.

Moreover, consider two portfolios $\pi_{k} \equiv\left(\mathscr{S}^{(k)}, \rho^{(k)}, v, \delta^{(k)}\right)$, for $k=1,2$, such that:

$$
\left\{\begin{array}{l}
\rho_{1}^{(1)} \geq \rho_{1}^{(2)} ; \\
\rho_{2}^{(1)}=\rho_{2}^{(2)} ; \\
\delta_{i \rightarrow j}^{(1)} \geq \delta_{i \rightarrow j}^{(2)}, \text { for } i=1, j=2 ; \\
\delta_{i \rightarrow j}^{(1)}=\delta_{i \rightarrow j}^{(2)}, \text { otherwise. }
\end{array}\right.
$$

Then the decision maker prefers portfolio $\pi_{1}$ to $\pi_{2}$.

4. Compound portfolios - Fix $n \in \mathbb{N}$. Consider $n$ portfolios $\pi_{k} \equiv$ $\left(\mathscr{S}^{(k)}, \rho^{(k)}, v^{(k)}, \delta^{(k)}\right)$, for $k=1,2, \ldots, n$, and a compound portfolio $\pi^{\star}$ having the $n$ portfolios as nodes as follows:

$$
\pi^{\star} \equiv\left(\left\{\pi_{1}, \pi_{2}, \ldots, \pi_{n}\right\}, \rho^{\star}, v^{\star}, \delta^{\star}\right) .
$$

Furthermore, define $\tilde{\pi} \in \mathbf{P}$ as $\tilde{\pi} \equiv(\widetilde{\mathscr{S}}, \tilde{\rho}, \tilde{v}, \tilde{\delta})$, where

$$
\widetilde{\mathscr{S}}=\bigcup_{k=1}^{n} \mathscr{S}^{(k)}
$$

two transformations $\phi$ and $\psi$ can be identified such that:

$$
\left\{\begin{array}{l}
\tilde{\rho}=\phi\left(\rho^{\star}, \rho^{(1)}, \rho^{(2)}, \ldots, \rho^{(n)}\right) \\
\tilde{\delta}=\psi\left(\delta^{\star}, \delta^{(1)}, \delta^{(2)}, \ldots, \delta^{(n)}\right)
\end{array}\right.
$$

and, for each $(i, j) \in \mathscr{S}^{\left(k_{i}\right)} \times \mathscr{S}^{\left(k_{j}\right)}$, we have

$$
\left\{\begin{array}{l}
\tilde{v}(i, j)=1 \text { if } k_{i} \neq k_{j} \text { and } v^{\star}\left(\mathbf{N}_{i}, \mathbf{N}_{j}\right)=1 ; \\
\tilde{v}(i, j)=1 \text { if } k_{i}=k_{j}=k \text { and } v^{(k)}(i, j)=1 ; \\
\tilde{v}(i, j)=0 \text { otherwise. }
\end{array}\right.
$$

Then, two transformations $\phi$ and $\psi$ as in (7) exist such that $\tilde{\pi}$ and $\pi^{\star}$ are equivalent for the preference order.

5. Independence - Consider two portfolios $\pi_{k} \equiv\left(\mathscr{S}^{(k)}, \rho^{(k)}, v^{(k)}, \delta^{(k)}\right)$, for $k=$ 1,2. Assume that $\mathscr{S}^{(1)}=\left\{X_{1}^{(1)}, \ldots, X_{n}^{(1)}\right\}$ and $\mathscr{S}^{(2)}=\left\{X_{1}^{(2)}, \ldots, X_{n}^{(2)}\right\}$, with $X_{i}^{(1)} \neq X_{i}^{(2)}$ for each $i=1, \ldots, n$. Suppose that $X_{1}^{(1)}>_{S D 1} X_{1}^{(2)}$ and suppose also that $\pi_{1}$ is preferred to $\pi_{2}$. Let us now consider two portfolios $\widetilde{\pi}_{1}, \widetilde{\pi}_{2}$ defined as follows:

$$
\tilde{\pi}_{k} \equiv\left(\tilde{\mathscr{S}}^{(k)}, \rho^{(k)}, v, \delta^{(k)}\right), \quad k=1,2,
$$


where $\tilde{\mathscr{S}}^{(1)}=\left\{\tilde{X}_{1}^{(1)}, X_{2}^{(1)}, \ldots, \tilde{X}_{n}\right\}$ and $\tilde{\mathscr{S}}^{(2)}=\left\{\tilde{X}_{1}^{(2)}, X_{2}^{(2)}, \ldots, \tilde{X}_{n}\right\}$, being $\tilde{X}_{1}^{(1)}$ $>_{S D 1} \tilde{X}_{1}^{(2)}$.

Then $\tilde{\pi}_{1}$ is preferred to $\tilde{\pi}_{2}$.

\section{References}

1. Abrahamsen, E.B., Aven, T.: On the consistency of risk acceptance criteria with normative theories for decision-making, Reliability Engineering \& System Safety 93(12), 1906-1910 (2008)

2. Castellano, R., Cerqueti, R.: Optimal consumption/investment problem with light stocks: A mixed continuous-discrete time approach, Applied Mathematics and Computation 218(12), 6887-6898 (2012)

3. Castellano, R., Cerqueti, R.: Mean-variance portfolio selection in presence of infrequently traded stocks, European Journal of Operational Research 234(2), 442-449 (2014)

4. Cerqueti, R., Lupi, C.: Risk measures on networks and expected utility, Reliability Engineering \& System Safety, 155, 1-8 (2016)

5. Cerqueti, R., Spizzichino, F.: Extension of dependence properties to semi-copulas and applications to the mean-variance model, Fuzzy Sets and Systems 220, 99-108 (2013)

6. Leippold, M., Trojani, F., Vanini, P.: A geometric approach to multiperiod mean variance optimization of assets and liabilities, Journal of Economic Dynamics and Control 28(6), 10791113 (2004)

7. Markowitz, H.: Portfolio selection, Journal of Finance 7(1), 77-91 (1952)

8. Markowitz, H.: Mean-variance approximations to expected utility, European Journal of Operational Research 234(2), 346-355 (2014)

9. Merton, R.C.: Lifetime portfolio selection under uncertainty: the continuous-time case, Review of Economics and Statistics 51, 247-257 (1969)

10. Merton, R.C.: Optimal consumption and portfolio rules in a continuous time model, Journal of Economic Theory 3, 373-413 (1971)

11. Rambaud, S.C., Perez, J.G., Sanchez Granero, M.A., Trinidad Segovia, J.E.: Markowitz's model with Euclidean vector spaces, European Journal of Operational Research 196, 1245$1248(2009)$

12. Scott J.: Social Network Analysis, 3rd Edition. Sage, London (2013)

13. Samuelson, P.A.: Lifetime portfolio selection by dynamic stochastic programming, Review of Economics and Statistics 51, 239-246 (1969)

14. Wasserman, S., Faust, K.: Social Network Analysis: Methods and Applications. Cambridge University Press, Cambridge (1994) 